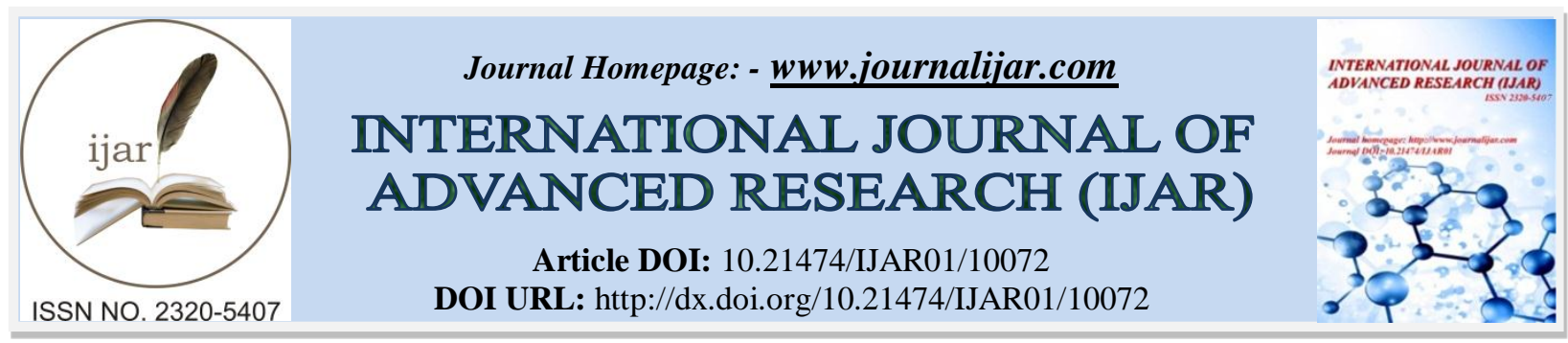

RESEARCH ARTICLE

\title{
CAUSE RARE DE RUPTURE UTERINE AU 2EME TRIMÈSTRE : PLACENTA ACCRETA SUR UTERUS SAIN.
}

\section{Afalah h., Kriouile K., Jayi S.,Fdili Alaoui F.Z., Chaara H.,El Fatemi H and Melhouf My A.} Service De Gynécologie Obstétrique Ii Chu Hassan Ii De Fes.

\section{Manuscript Info}

Manuscript History

Received: 12 September 2019

Final Accepted: 14 October 2019

Published: November 2019

\begin{abstract}
Généralités: Le placenta accréta est une affection obstétricale rare, qui se rencontre principalement au troisième trimestre et pouvant mener à des complications mortelles.

Observation: nous rapportons un cas de rupture utérine survenue chez une patiente de $41 \mathrm{ans}$, grande multipare qui a consulté dans un tableau d'instabilité hémodynamique et d'abdomen aigu sur grossesse de 20 $\mathrm{SA}$, avec à l'échographie un fœtus en intra abdominale sans activité cardiaque et un hémopéritoine de grande abondance. Une laparotomie d'urgence a objectivé une rupture utérine avec placenta inccréta pour lequel la patiente a subi une hystérectomie totale.

Conclusion: Bien que rare au $2^{\text {ème }}$ trimestre, la rupture utérine sur placenta accréta doit être suspectée devant un tableau de rupture utérine, et doit surtout etre recherché au cours du suivi prénatal notamment chez les patientes à risque à fin d'améliorer le pronostic à travers une organisation de la prise en charge
\end{abstract}

Copy Right, IJAR, 2019,. All rights reserved.

\section{Introduction:-}

Le placenta accréta est une adhérence anormale du placenta au myomètre, du fait de l'absence localisée ou diffuse de la caduque basale [1]. Son incidence est en augmentation avec celle du taux de césarienne. Il est associé à une morbidité et une mortalité maternelle importante du fait d'hémorragies de la délivrance pouvant être graves, rupture utérine et l'envahissement des organes voisins [2]. Et la rupture utérine spontanée au cours de $2^{\text {ème }}$ trimestre de la grossesse est une complication très rare mais possible [3]. Nous présentons ici un cas intéressant d'un placenta accréta qui a conduit à une rupture utérine au deuxième trimestre nécessitant une hystérectomie.

\section{Observation:-}

Patiente âgée de 41 ans, grande multipare G13P11,sans antécédants particulier, notamment pas d'antécédants d'utérus cicatriciel, admise aux urgences de maternité pour prise en charge des douleurs abdomino-pelviennes d'installation brutale évoluant depuis 3 jours chez qui l'examen a trouvé un pouls à $120 \mathrm{~b} / \mathrm{min}$, pression artérielle à 90/60 $\mathrm{mmHg}$, sueurs profuses, conjonctives décolorées, hémoglobine à $5 \mathrm{~g}$ ), l'examen obstétrical a objectivé un abdomen distendu douloureux dans son ensemble, bruits cardiaques fœtaux non perçus. L'échographie obstétricale Après mise en condition de la patiente a révélé une grossesse monofoetale non évolutive, foetus en intra-abdominal avec signes de mort ancienne, présence d'une rupture utérine au niveau fundique associée à un épanchement intra péritonéale de grande abondance. Une laparotomie d'urgence a été réalisée chez notre patiente avec à l'exploration un hémopéritoine de grande abondance aspiré estimé à $2500 \mathrm{ml}$, découverte d'un fotus en intra abdominale de sexe

Corresponding Author:-Afalah h.

Address:-Service De Gynécologie Obstétrique Ii Chu Hassan Ii De Fes. 
féminin faisant 20 SA (Figure 1), après clampage et section du cordon et extraction du foetus, on note une perforation utérine postéro-fundique en regard du placenta qui infiltre la paroi jusqu'à la séreuse utérine faisant évoquer un placenta inccréta, le reste de l'exploration était sans particularités, une hystérectomie inter-annexielle d'hémostase a été réalisée, patiente a été transfusée en per opératoire par 4 CG+6PFC

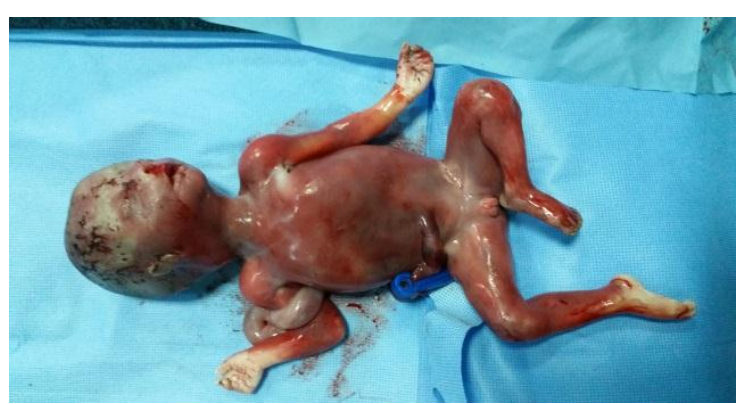

Figure 1:-mort né sexe féminin faisant 20 SA (service de gynécologie obstétrique II CHU Hassan II)

Les suites post opératoires ont été marquées par une infection de la paroi jugulée par l'antibiothérapie et les soins biquotidiens. Patiente déclarée sortante à j+17 du post opératoire.

L'utérus (figure 2) a été envoyé pour étude anatomopathologique dont l'aspect histologique compatible avec un placenta accréta (figure3) avec des lésions de chorioamniotite.

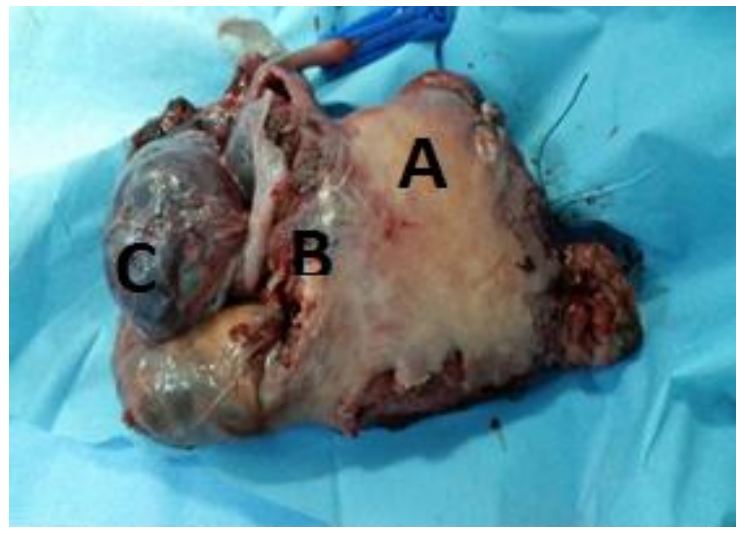

Figure 2:-pièce d'hysterectomie interannexielle avec le placenta sur place

1. A : face post de l'utérus

2. B :rupture fundique

3. C: placenta adhérent au myomètre + séreuse

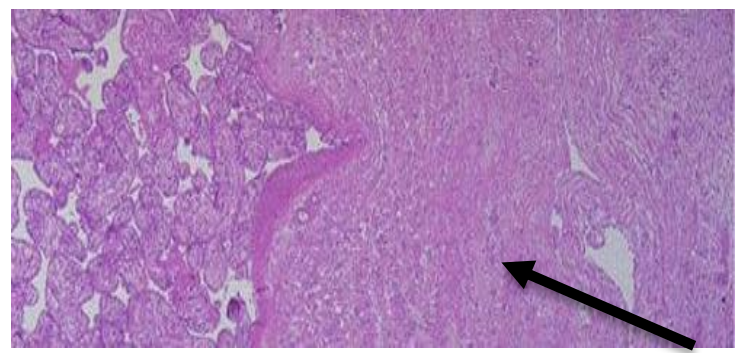

Figure 3 :-aspect histologique d'un placenta accreta: Les villosités choriales envahissent directement le myomètre. (Laboratoire d'anatomopathologie CHU Hassan II)

flèche :limite entre villosité et myomètre 


\section{Discussion:-}

L'incidence du placenta accréta ne cesse d'augmenter ces dernières années. Cette progression semble directement corrélée à l'augmentation des altérations de la muqueuse utérine au cours de la vie génitale des patientes, et plus particulièrement à l'augmentation croissante du taux de césariennes. Elle devient plus fréquente lorsque le placenta est bas inséré son incidence dans ce cadre étant estimée à environ $10 \%$ [2].

La Rupture spontanée de l'utérus due au placenta accréta est l'une des complications graves entraînant une mortalité élevée qui est plus fréquemment observée au troisième trimèstre et est très rare au deuxième trimestre ce qui souligne l'intérêt de notre travail [3].

L'étiologie précise de placenta accréta est inconnue, mais existent des facteurs de risque, car tout déficit quantitatif ou qualitatif de la décidua crée une zone propice à une invasion non contrôlée du trophoblaste et donc à la survenue d'un placenta accréta [8].

Cette anomalie de la décidua survient à la suite des lésions de la muqueuse endométriale, elles mêmes secondaires à des séquelles d'endométrite chronique du post-partum ou du post-abortum, ou à des cicatrices traumatiques de l'utérus dont les plus importantes sont la césarienne, le curetage utérin et les myomectomies [5]. Dans la plupart des cas, le placenta accréta est une combinaison de plusieurs facteurs et il est peu probable de l'attacher à un seul facteur [4]. On distingue trois types de placenta accréta. le placenta accreta proprement dit ou les villosités sont en contact du myomètre et pénètrent plus ou moins profondément dans le myomètre; le placenta increta: les villosités envahissent le myomètre et le placenta percreta dont les villosités dépassent le myomètre, envahissant parfois les organes voisins (vessie) [4-5].

Contrairement à de nombreux rapports, où un placenta accréta a été diagnostiqué chez des patientes porteuses d'un utérus cicatriciel [5], notre patiente n'avait pas de notion de césarienne précédente. Cependant, il y'avait d'autres facteurs comme l'âge maternel avancé et la multiparité pouvant être en cause. La rupture utérine spontanée au début de la gestation est extrêmement rare [6]. Les cas de rupture utérine sur placenta accréta au début de la grossesse au ont été décrits entre la $10^{\text {ème }}$ et la $20^{\text {ème }}$ semaine de gestation, mais parmi eux seuls quelques cas ont été rapportés sur utérus sain [9].

La clinique est souvent silencieuse au cours de la grossesse. Le diagnostic est en général suspecté devant des facteurs de risques et exploré par l'échographie est devenue l'outil de dépistage principal pour les femmes à risque de placenta accréta, et l'imagerie de Résonnance Magnétique (IRM) [14-16].

Les signes échographiques classiquement décrites sont la présence de lacunes intraplacentaires, l'absence d'un liseré hypoéchogène entre le placenta et le myomètre, une interruption de la zone hyperéchogène à l'interface de la séreuse utérine et de la vessie, et la présence d'un aspect pseudotumoral du placenta en regard de la séreuse utérine [10-14].

L'IRM a pu etre rapporté comme étant utile au diagnostic, parmi les critères diagnistiques proposés figurent un bombement anormal du ségment inférieur, une hétérogéneité de l'intensité du signal du placenta en T2, des bandes noires intraplacentaires en T2 [15].

Le diagnostic est parfois diagnostiqué au moment de la délivrance ou comme notre cas par un tableau d'hémorragie interne. Cette complication est certe rare mais grave soulignant le grand intérêt du diagnostic préopératoire de cette entité [9].

certains diagnostics différentiels peuvent poser problème au cours de la délivrance comme le placenta incarcéré conservé, le choriocarcinome et c'est l'étude anatomo pathologique qui tranche [7].

La prise en charge est basée sur la césarienne programmée à 34 semaines d'aménorhée avec une hystérectomie qui est considérée comme le "gold standard », et consiste à réaliser une hystérectomie après la naissance de l'enfant sans tentative de délivrance artificielle quand le diagnostic prénatal de placenta accreta a été réalisé, ou après une tentative de délivrance artificielle quand le diagnostic de placenta accreta est effectué en peropératoire [10]. Cette option pourrait réduire la morbidité maternelle [11], mais elle résulte nécessairement en la perte de la fertilité de la patiente.

le traitement conservateur a été rapporté par certains auteurs suivie d'administration de méthotréxte [7], qui semble une option potentiellement intéressante pour les patientes ayant un désir ultérieur de grossesses. Il consiste à extraire 
l'enfant, à nouer puis couper le cordon ombilical à sa base, à laisser en place in situ le placenta qui adhère partiellement ou totalement au myomètre, après soit une tentative prudente de délivrance manuelle, soit aucune tentative de délivrance manuelle $[1,6]$. Cette option présente comme principal avantage de potentiellement préserver la fertilité des patientes [12-13]. Cependant, cette option reste actuellement très controversée car le traitement conservateur expose les patientes à un risque d'infection abdomino-pelvienne voire de péritonite, ainsi que d'hémorragie massive en post-partum.

\section{Conclusion:-}

le placenta accréta est une pathologie à risque de complications hémorragiques graves au cours de la grossesse et du post-partum. A travers notre cas et à la lumière d'une revue de la litérature nous attirons l'attention des praticiens sur l' intérêt de penser au placenta accréta au cours du suivi prénatal encore plus chez les femmes à risque ce qui permettrait d'améliorer le Pronostic de ces patientes en organisant une PEC adaptée et en présence d'une équipe multidisciplinaire.

\section{References:-}

1. Konan Blè R,Adjoussou $\mathrm{S}$, Doukoure $\mathrm{B}$ et al. une étiologie particulière de perforation utérine spontanée au deuxième trimestre de grossesse : le placenta accréta.gynecol Obstet Fertil 2011;39:e11-4.

2. Melhouf $\mathrm{A}$,Rhajdi A. placenta accréta, à propos de 10 cas. thèse pour obtention du doctorat en médecine. $\mathrm{N}^{\circ} 107 / 10$.faculté de médecine et de pharmacie de Fès.

3. H.El Mhabrech,A.Zrig.A Ben Salem et al.cause rare de rupture utérine spontanée au premier trimèstre de grossesse:le placenta percreta étude de cas 2013.

4. Bourgeois JM.Tout ce que vous avez voulu savoir sur le placenta accreta-increta-percreta.ultrasound Obstet Gynecol $2005 ; 26: 89-96$

5. Placenta accreta. Encycl Méd Chir, Obstétrique, 5-069-A-30, 2003, 11 p.

6. Y. Gielchinskya, N. Rojansky, S. J. Fasouliotis and Y. Ezra Placenta Accreta-Summary of 10 Years: A Survey of 310 Cases placenta (2002), 23, 210-214

7. Attieh E, AbboudJ, ChalhoubS, RiachiM. Placenta percreta et rupture utérine. À propos de deux cas. J Gynécol Obstét Biol Reprod 1993 ; $22: 649-652$.

8. Hung TH, Shau WY, Hsieh CC, Chiu TH, Hsu JJ, Hsieh TT. Risk factors for placenta accreta. Obstet Gynecol1999; 93 :545-550.

9. Comstock CH, Love Jr. JJ, Bronsteen RA, et al. Sonographic detection of placenta accreta in the second and third trimesters of pregnancy. Am J Obstet Gynecol 2004;190:1135-40.

10. Extrait des Mises à jour en Gynécologie et Obstétrique - Tome XXXIII publié le 9.12.2009

11. Clark SL, Belfort MA, Dildy GA, Herbst MA, Meyers JA, Hankins GD. Maternal death in the 21st century: causes, prevention, and relationship to cesarean delivery. Am J Obstet Gynecol 2008;199:36.e1-5.

12. Kayem G, Pannier E, Goffinet F, Grange G, Cabrol D. Fertility after conservative treatment of placenta accreta. Fertil Steril 2002;78:637-8.

13. Alanis M, Hurst BS, Marshburn PB, Matthews ML. Conservative management of placenta increta with selective arterial embolization preserves future fertility and results in a favorable outcome in subsequent pregnancies.Fertil Steril 2006;86:1514.e3-7

14. G.Kayem, H.Keita . prise en charge des placentas preavia et accréta journal de gynécologie obstétrique et biologie de la reproduction 2014

15. Lax A, Prince MR, Mennitt KW, Schwebach JR, Budorick NE. The value of specific MRI features in the evaluation of suspected placental invasion. Magn Reson Imaging 2007;25:87—93 [Epub 2007/01/16].

16. Eric R.M. Jauniaux Amar Bhide et Jason D. Wright Obstetrics: Normal and Problem Pregnancies, Chapter 21, 456-466 @ 2017. 